\title{
Formation of a sandy near-bed transport layer from a fine-grained bed under oscillatory flow
}

\author{
Hanzhuang Liang, ${ }^{1}$ Michael P. Lamb, ${ }^{2}$ and Jeffrey D. Parsons ${ }^{3}$ \\ Received 13 April 2006; revised 13 September 2006; accepted 4 October 2006; published 6 February 2007.
}

[1] Bed surface coarsening was found to be an important effect for the formation of ripples and the dynamics of the boundary layer above a predominantly silt-sized sediment bed (median particle size equal to $26 \mu \mathrm{m}$; 20\% fine sand, $70 \%$ silt, $10 \%$ clay) under oscillatory flow (with orbital velocities of $0.32-0.52 \mathrm{~m} / \mathrm{s}$ ) in a laboratory wave duct. Following bed liquefaction, substantial winnowing of the bed surface occurred due to entrainment of finer material into suspension. Bed surface coarsening was quantified with micro-scale visualization using a CCD (charged-coupled device) camera. Under most wave orbital velocities investigated, the coarse surface particles were mobilized as a nearbed transport layer approximately 4 grain-diameters thick. The transport of these coarse sediments ultimately produced suborbital or anorbital ripples on the bed, except for the highest orbital velocities considered where the bed was planar. Micro-scale visualizations were used to construct a maximum (particle) velocity profile extending through the near-bed transport layers using particle-streak velocimetry (PSV). These profiles had a distinctive kink in log linear space at the height of the transport layer, suggesting that the near-bed sediment transport reduced skin friction and contributed to the boundary roughness through extraction of momentum.

Citation: Liang, H., M. P. Lamb, and J. D. Parsons (2007), Formation of a sandy near-bed transport layer from a fine-grained bed under oscillatory flow, J. Geophys. Res., 112, C02008, doi:10.1029/2006JC003635.

\section{Introduction}

[2] The continental shelf is an extremely dynamic region where both wind-generated surface waves and currents are capable of mobilizing seafloor sediments. Continental shelves possess a variety of grain sizes, often with sandsized sediment near shore (on the inner shelf) and finer material further seaward in "mid-shelf mud deposits" [Wright and Nittrouer, 1995; Crockett and Nittrouer, 2004]. Mud and sand can migrate cross-shore due to currents, wave asymmetry, and gravity-driven processes [e.g., Wright et al., 2002; Styles and Glenn, 2005]. This transport is often highly intermittent and causes extremely high near-bed sediment concentrations when high transport occurs [Ogston et al., 2000; Traykovski et al., 2000; Wright et al., 2002]. It is during these periods when the seafloor is most profoundly shaped [Scully et al., 2003].

[3] The entrainment and transport of sand under waves has received considerable research attention through field, laboratory, and theoretical means. During low transport stages, ripples form on a sandy seabed [e.g., Wiberg and Harris, 1994]. At higher transport stages, ripples are

\footnotetext{
${ }^{1}$ School of Oceanography and Applied Physics Laboratory, University of Washington, Seattle, Washington, USA.

${ }^{2}$ Department of Earth and Planetary Sciences, University of California, Berkeley, California, USA.

${ }^{3}$ School of Oceanography, University of Washington, Seattle, Washington, USA.
}

Copyright 2007 by the American Geophysical Union. 0148-0227/07/2006JC003635\$09.00 washed out and sand is transported in a thin $(<1 \mathrm{~cm})$ near-bed transport layer or sheet flow [e.g., Flores and Sleath, 1998; Dohmen-Janssen et al., 2001]. The most widely cited wave-boundary layer models were developed using a time-invariant eddy viscosity to close the equations of motion [i.e., Grant and Madsen, 1979, 1982, 1986] (hereafter referred to as "GM"). Ripples and sheet flow are typically modeled as an additional roughness on the flow [Grant and Madsen, 1982], which therefore increases velocity gradients and shear velocity in the bulk of the boundary layer, although more complex multiphase models exist [Foda, 2003; Hsu and Hanes, 2004]. The predictions of shear velocity can then be used to drive sediment suspension models [e.g., García and Parker, 1991].

[4] Historically, much less attention has been given to large-scale wave motions (that produce a turbulent wave boundary layer) over finer grained beds of silt or clay. Recent oceanographic observations, however, have shown that highly concentrated $(>10 \mathrm{~g} / \mathrm{l})$, relatively thin $(\sim 10 \mathrm{~cm})$, benthic suspensions or fluid muds occur frequently on continental margins with fine-grained beds during large storm waves [e.g., Ogston et al., 2000; Traykovski et al., 2000]. Several adaptations have been proposed to account for sediment stratification in combined current and wave boundary layer models [Wiberg and Smith, 1983; Glenn and Grant, 1987; Styles and Glenn, 2000], but these have yet to be extended to the wave boundary layer in a rigorous manner because incorporating a time-dependent stratification correction is difficult [Styles and Glenn, 2000]. Some simple bulk-averaged models have been developed for fine- 


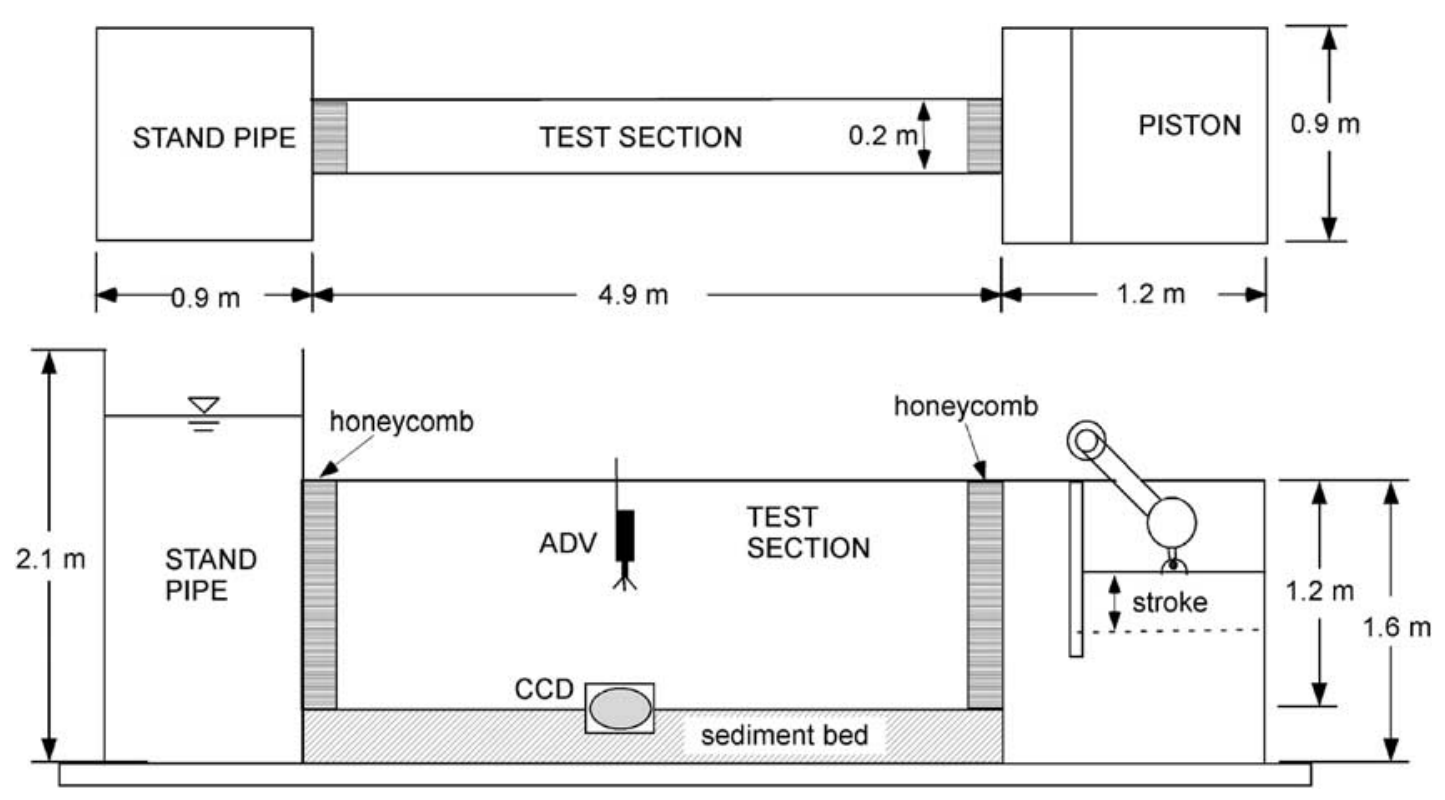

Figure 1. Schematic of the experimental wave tank in (top) plan view and (bottom) side view.

grained suspensions [e.g., Wright et al., 2001], but evaluation of these have been limited because detailed field measurements of velocity, sediment concentration, and particle size within centimeters above the bed are difficult.

[5] Recently, experiments were conducted on the interactions of wave orbital motions with a predominantly siltsized sediment bed [Lamb et al., 2004; Lamb and Parsons, 2005] (herein referred to as LDP). LDP showed that significant amounts of sediment were entrained into the water column under modest orbital velocities. Stratification from the suspended sediment limited vertical mixing of momentum and consequently reduced the size of wave boundary layer from that in sediment-free conditions, often to less than $3 \mathrm{~mm}$. Despite the reduction in boundary layer size, turbulent energy was diffused high into the water column where it supported dense-suspensions (i.e., highdensity suspensions) centimeters thick. Finer sediment was preferentially transported into the upper water column, such that the near-bed percentage of suspended sand was high (up to $78 \%$ ), despite the fact that the initial bed only contained about 20\% fine sand (with the remainder being $70 \%$ silt and $10 \%$ clay). Coarsening of the bed surface through winnowing processes allowed for the formation of ripples and potentially limited entrainment of finer sediment through armoring. Unfortunately, the instrumentation used by LDP (acoustic Doppler velocimeter (ADV) and siphons for flow sampling) did not have the necessary resolution to investigate the highly dynamic, near-bed region $(<3 \mathrm{~mm}$ above the bed) where entrainment, bed-surface coarsening, ripple formation, vertical mixing of momentum, and production of turbulent kinetic energy occurred.

[6] Herein we present high resolution, sub-millimeterscale visualizations of this near-bed region in laboratory experiments similar to those of LDP. These visualizations are used to quantitatively document coarsening of the bed surface in response to entrainment of finer particles into the water column. The sand-sized particles transported near the bed eventually formed ripples under most conditions inves- tigated. Sand particles were transported across ripple crests in thin $(\sim 0.35 \mathrm{~mm})$, near-bed transport layers, akin to sheet flows in coarser-grained environments. Particle streak velocimetry (PSV) was used to construct particle velocity profiles through the near-bed transport layers.

[7] The term sheet flow is typically used to describe highly concentrated near-bed transport layers that occur during high transport conditions under waves when ripples are washed out and the bed is planar. Within this layer, interactions between the particles, fluid and bed are all probably important. For most of the experiments reported here, we documented such a layer, but when the bed surface contained ripples. It has previously been recognized that such transport layers can exist and are an important roughness effect even when the bed is rippled [e.g., Grant and Madsen, 1982; Wiberg and Rubin, 1989]. Near-bed transport layers also have been recognized to be important in aeolian [Bagnold, 1941; Owen, 1964; Anderson and Hallet, 1986; McEwan, 1993; Bauer et al., 2004] and fluvial [Smith and McLean, 1977; Dietrich, 1982; Gust and Southard, 1983; Best et al., 1997; Bergeron and Carbonneau, 1999; McEwan et al., 1999] sediment transport, and they are sometimes called saltation or bed load layers. In order to avoid confusion with the term sheet flow and not to imply a transport process, herein we refer to these as near-bed transport layers.

\section{Experimental Setup and Procedure}

[8] The experimental facility used in our experiments is the same used and described in detail by LDP. Here, relevant points will be summarized. The piston-driven U-tube (Figure 1) produces near sinusoidal oscillatory motions with orbital velocities and orbital diameters comparable to conditions near the seabed on continental shelves under wind-driven surface gravity waves. Velocity fluctuations due to advected turbulence from the end tanks are held to less than $10 \%$ of the orbital motions in the test section through the use of plastic honeycomb [LDP]. The side-wall 
Table 1. Experimental Conditions

\begin{tabular}{|c|c|c|c|c|c|c|c|c|}
\hline & \multicolumn{8}{|c|}{ Experiment } \\
\hline & 1 & 2 & 3 & 4 & 5 & 6 & 7 & 8 \\
\hline$U_{o r b}, \mathrm{~m} / \mathrm{s}$ & 0.22 & 0.32 & 0.37 & 0.39 & 0.44 & 0.47 & 0.52 & 0.55 \\
\hline$d_{0}, \mathrm{~m}$ & 0.43 & 0.43 & 0.7 & 0.7 & 0.7 & 0.7 & 0.7 & 0.7 \\
\hline$T, \mathrm{~s}$ & 6 & 4.23 & 5.94 & 5.64 & 5 & 4.68 & 4.23 & 4 \\
\hline Initial bed ${ }^{\mathrm{a}}$ & new & new & new & new & new & after exp. 5 & new & after exp. 4 \\
\hline Bedform $^{\mathrm{b}}$ & plane & Sub- & an- & an- & an- & an- & an- & plane \\
\hline$\lambda, \mathrm{mm}$ & $\mathrm{n} / \mathrm{a}$ & 61 & 63.5 & 57.2 & 63.5 & 88.9 & $>88.9$ & $\mathrm{n} / \mathrm{a}$ \\
\hline$\eta, \mathrm{mm}$ & $\mathrm{n} / \mathrm{a}$ & 6.35 & 5.56 & 4.76 & 4.76 & 3.18 & 1.59 & $\mathrm{n} / \mathrm{a}$ \\
\hline $\bar{D}, \mu \mathrm{m}$ & 26 & 87 & 82 & 92 & 100 & 100 & 93 & 83 \\
\hline$h_{E}, \mathrm{~mm}$ & $<1$ & 6.4 & 4.8 & 8 & 8.7 & $\mathrm{n} / \mathrm{a}$ & 9.5 & $\mathrm{n} / \mathrm{a}$ \\
\hline$h_{S}, \mathrm{~mm}$ & 0.00 & 0.17 & 0.18 & 0.20 & 0.18 & 0.18 & 0.14 & 0.15 \\
\hline$h_{T}, \mathrm{~mm}$ & 0.00 & 0.34 & 0.44 & 0.45 & 0.35 & 0.38 & 0.24 & 0.25 \\
\hline$C_{T}$ & 0.00 & 0.30 & 0.25 & 0.27 & 0.31 & 0.28 & 0.35 & 0.36 \\
\hline$u_{* a}, \mathrm{~cm} / \mathrm{s}$ & $?$ & $?$ & 3.5 & 3.0 & 2.0 & 2.8 & 2.6 & 5.6 \\
\hline$u_{* b}, \mathrm{~cm} / \mathrm{s}$ & $?$ & $?$ & 2.0 & 1.3 & 1.9 & 2.4 & 2.0 & 2.2 \\
\hline$z_{0 a}, \mathrm{~mm}$ & $?$ & $?$ & 0.18 & 0.091 & 0.035 & 0.080 & 0.065 & 0.15 \\
\hline$\underline{z_{0 b}, \mathrm{~mm}}$ & $?$ & $?$ & 0.026 & 0.011 & 0.022 & 0.030 & 0.020 & 0.021 \\
\hline
\end{tabular}

${ }^{a}$ Experiments 6 and 8 were performed 30 min after experiments 5 and 4, respectively. All other experiments had a new, well-mixed bed of sediment.

b plane, planar bedding; sub-, suborbital ripple with $20<d_{0} / \eta<100$; an-, anorbital ripple with $d_{0} / \eta>100$, following Wiberg and Harris [1994]. Question mark indicates that data were not collected.

boundary layers are $<10 \%$ of the interior width of the test section $(20 \mathrm{~cm}$; Figure 1$)$ and therefore do not significantly influence the orbital motions in the test section. The flume walls are Plexiglas, which allow for detailed visual observations and measurements.

[9] A total of eight experiments were completed in the experimental wave tank, in which the maximum free-stream wave orbital velocity $U_{\text {orb }}$ ranged from $0.22-0.32 \mathrm{~m} / \mathrm{s}$ with a wave orbital diameter $d_{0}=0.43 \mathrm{~m}$ and $U_{o r b}=0.37-$ $0.55 \mathrm{~m} / \mathrm{s}$ with $d_{0}=0.70 \mathrm{~m}$ (Table 1 ). The wave orbital diameter $d_{0}$ is defined as $d_{0}=U_{\text {orb }} T / \pi$, where $T$ is the freestream wave period. The experiments are numbered according to increasing $U_{\text {orb }}$ (Table 1). The free-stream orbital motions were measured in the center of the test section, approximately $15 \mathrm{~cm}$ above the sediment bed (well above the bottom boundary layer), using a micro acoustic-Doppler velocimeter (ADV).

[10] Before an experiment, sediment was mixed thoroughly with fresh tap water to make a paste and poured into the experimental duct, so that the initial bed was approximately $0.15 \mathrm{~m}$ thick. The sediment bed then was raked flat and the flume was filled with fresh tap water. Some of the finest sediment was suspended into the water column as the duct was filled with water, and this sediment later settled forming a thin $(\sim 1 \mathrm{~mm})$ cap on top the sediment bed. These fine sediments were immediately suspended following the commencement of oscillatory motions and did not affect the initial well-mixed state of the sediment bed. The sediment was composed of crushed silica silt with a median grain size $D_{50}$ of approximately $26 \mu \mathrm{m}$ (Figure 2). The mixture contained $\sim 10 \%$ clay $(D<$ $3.9 \mu \mathrm{m})$ and $20 \%$ fine sand $(D>63 \mu \mathrm{m})$, where $D$ denotes the particle diameter. The mineralogical composition was $99.7 \%$ crushed angular silica, which appeared white. The remaining $0.3 \%$ contained a variety of oxidized species, some of which appeared black when viewed next to the silica. These darker impurities made for ideal tracers.

[11] A newly mixed batch of sediment was set in place for each experiment except for experiments 6 and 8. These two experiments were run approximately $30 \mathrm{~min}$ after experi- ments 5 and 4, respectively. Experiments 6 and 8 had higher orbital velocities than experiments 5 and 4 (Table 1), and the deposits and bedforms inherited from these previous experiments were easily reworked.

[12] Macroscopic video was taken during each experiment and used to measure ripple dimensions and to document the phase of the wave during microscopic visualization.

\subsection{Micro-Scale Visualization}

[13] The velocity of particles near the bed was estimated using particle-streak velocimetry (PSV). PSV consists of measuring the length of a streak left on film from a moving particle when a camera shutter is left open for a fixed amount of time. We used a high resolution CCD (chargedcoupled device) camera to obtain images at $30 \mathrm{~Hz}$ with

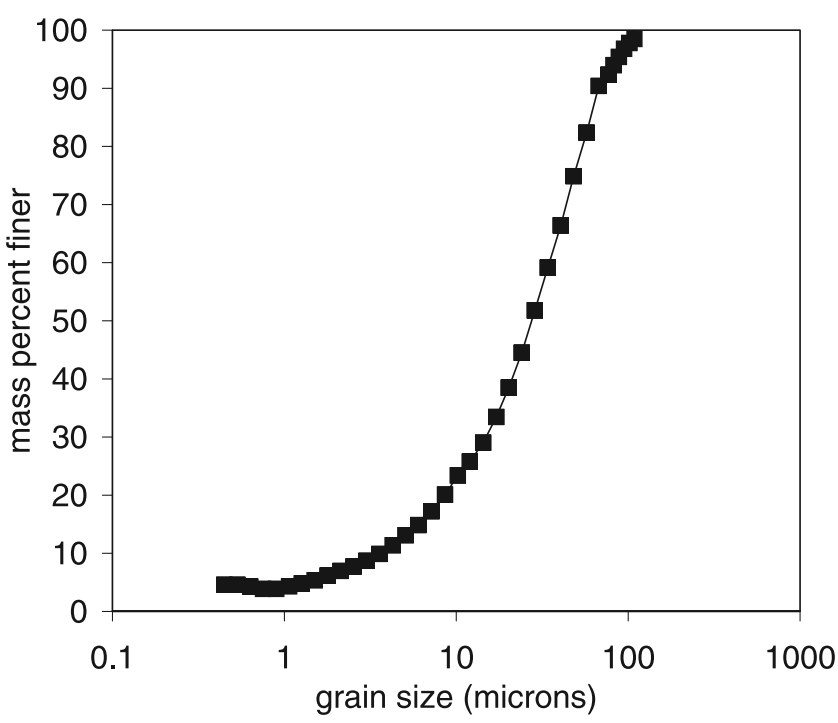

Figure 2. Grain-size distribution of the initial sediment bed mixture. The sample was split with a $63 \mu \mathrm{m}$ sieve and the coarse fraction was measured with a settling column and the fine fraction was measured with a Micrometrics Sedigraph 5100. 

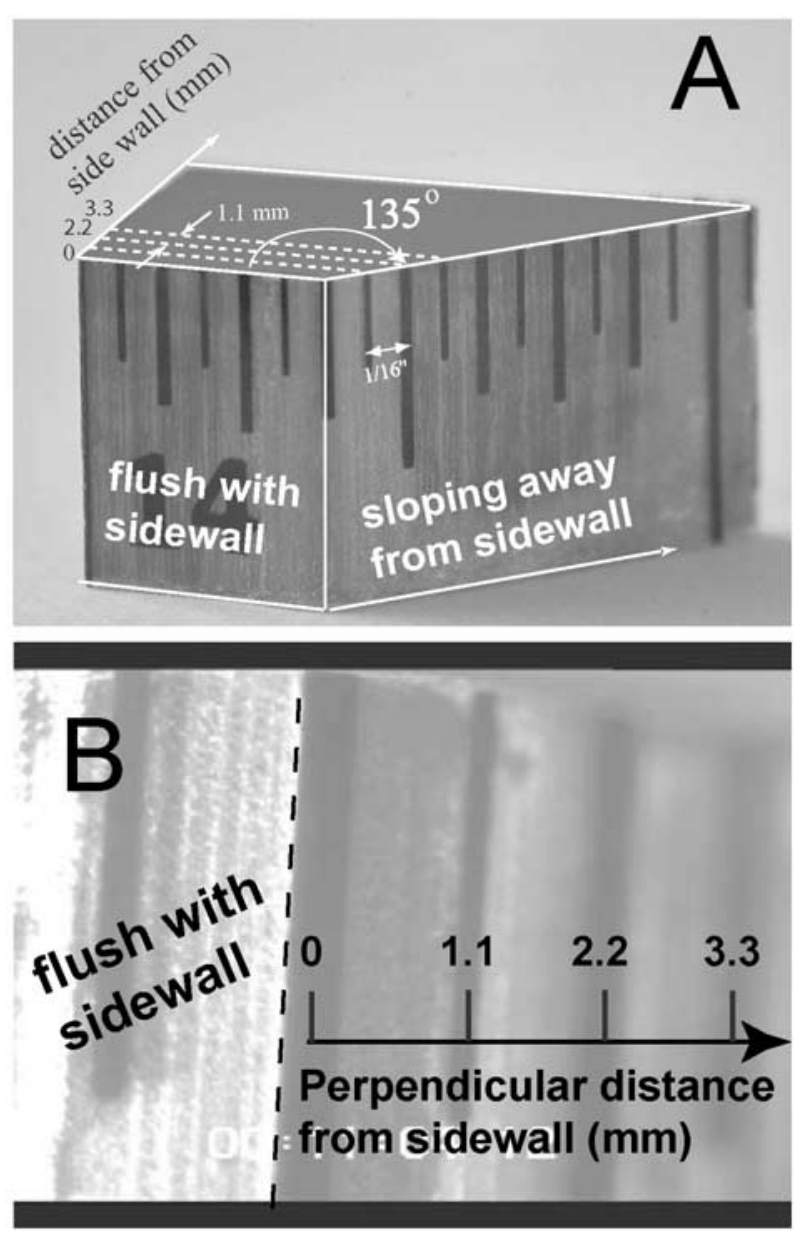

Figure 3. (a) Photograph of measurement prism used to estimate the distance of particles from the sidewall of the flume. The measuring tape had increments of $1.6 \mathrm{~mm}$ (1/16 in.) and was mounted to two faces of a rectangular prism with a $135^{\circ}$ vertex. One face was fixed flush to the sidewall of the wave duct and the other protruded away from the wall into the experimental test section. After correcting for the angle, each increment on the measuring tape was equivalent to $1.1 \mathrm{~mm}$ in the plane perpendicular to sidewall. (b) View of the prism through the CCD camera with the field of view set so that distance from the sidewall became progressively blurred. Blurry particle streaks were chosen to measure particle velocities to ensure that they were greater than about $2 \mathrm{~mm}$ from the wall.

exposure times of either 0.004 or $0.008 \mathrm{~s}$. These exposure times were chosen to ensure that the streaks were fully captured with respect to the field of view and focal depth. We used light from either a slide projector or a laser point to visualize the particles.

[14] The camera was positioned so that it was looking through the clear Plexiglas wall of the wave-duct into the test section, and was near the level of the sediment bed (Figure 1). Care was taken to insure that the particles measured were far enough from the sidewall of the duct so that they were not significantly influenced by the wall boundary layer. A measuring tape with $1.6 \mathrm{~mm}(1 / 16 \mathrm{in}$.) markings was mounted onto a rectangular prism, and this was inserted into the test section and placed flush against the sidewall (Figure 3). Because the vertex of the prism made a $135^{\circ}$ angle with the sidewall, the markings on the measuring tape indicated successive $1.1 \mathrm{~mm}$ distances perpendicular from the sidewall. The CCD camera was focused on the front face of the prism with a field of view of $4.8 \times 6.4 \mathrm{~mm}$ and a focal depth ranging from 1.0 to $1.5 \mathrm{~mm}$. As shown in Figure 3b, the angled face became incrementally blurred under these settings. This blurriness was used as a proxy for distance from the sidewall. Objects within $1.1 \mathrm{~mm}$ of the sidewall were sharp and well focused. Objects were slightly blurry at $2.2 \mathrm{~mm}$ from the wall. Objects farther than $5 \mathrm{~mm}$ from the sidewall were indistinguishable.

[15] The goal of these measurements was to measure the maximum particle velocity. Thus, we took pictures when the flow was moving the fastest and selected streaks from those images that were the longest (Figure 4). We also selected streaks that were the blurriest, while still distinguishable, to guarantee that they were at least $2 \mathrm{~mm}$ away from the wall. Most often trace impurities were used to record velocities as they provided ideal tracers (Figure 4). The distance from the immobile bed $z$ and the length of the particle streak $d l$ were measured from the images and used to calculate the maximum particle velocity profile by $u(z)=d l / d t$, where $d t$ is the exposure time for each still image.

[16] The measured particle velocities might underestimate the maximum particle velocity if the local velocity was not at its peak value (i.e., the phase of the wave was not at its maximum value) when the picture was taken or if the target particle was within the sidewall boundary layer. All of the particles measured were $2-5 \mathrm{~mm}$ from the sidewall. Unfortunately, we could not measure the thickness of the sidewall boundary layers. We did, however, measure the bottom boundary layer thickness to be less than $4 \mathrm{~mm}$ using the ADV when the sediment bed was replaced by a smooth Plexiglas bed (i.e., the same material that composes the sidewalls) and the maximum orbital velocity was $50 \mathrm{~cm} / \mathrm{s}$ and the wave period was 3.1 s (i.e., conditions similar to those used in the sediment experiments herein). Flores and Sleath [1998] also reported that the orbital velocity reached $U_{\text {orb }}$ around $3 \mathrm{~mm}$ away from the sidewall from comparable waves in a similar wave duct. The bottom boundary layer model of GM yields a boundary layer thickness of $\sim 15 \mathrm{~mm}$ for hydraulically smooth flow under wave conditions similar to these experiments. Using this larger value of $15 \mathrm{~mm}$, the orbital velocity at $z=2 \mathrm{~mm}$ would be $\sim 25 \%$ smaller than $U_{\text {orb }}$ (according to orbital velocity profiles calculated from GM).

\subsection{Bed Surface Grain Size}

[17] The grain size of the bed surface was estimated from the CCD photographs taken during the experiments. Unfortunately, image resolution was not sufficient to allow for counting and measurement of all of the particles. Instead, we counted and measured just the coarser particles on the bed surface that were identifiable. The mean grain size of the bed surface can be estimated roughly from

$$
\bar{D}=\frac{L}{n}
$$



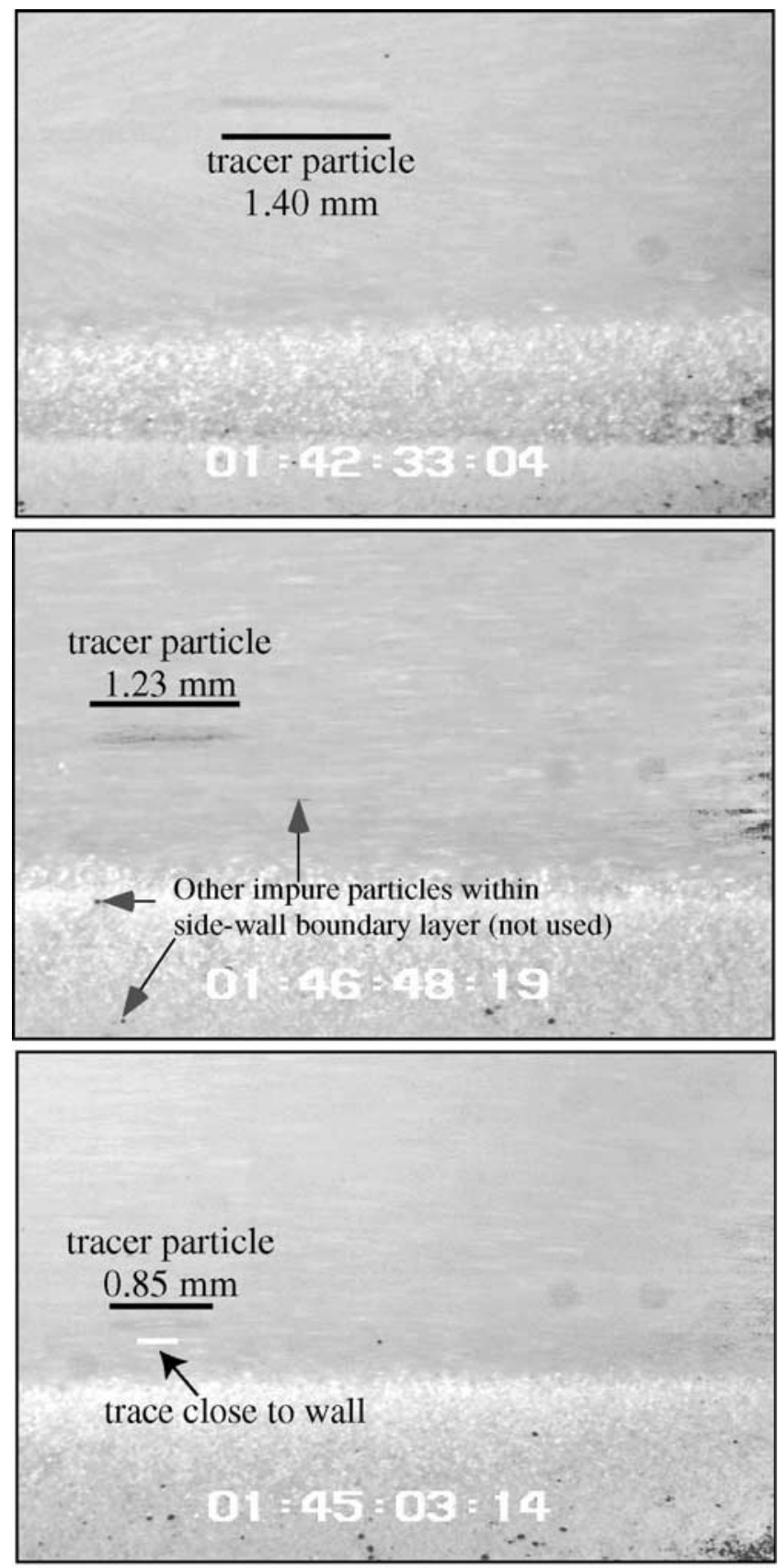

Figure 4. Three examples of particle-streak velocimetry (PSV) from experiment 8 where $U_{\text {orb }}=0.55 \mathrm{~m} / \mathrm{s}$ and $T=4 \mathrm{~s}$. Exposure time for each image was $d t=0.004 \mathrm{~s}$. Black particles were oxidized impurities. White particles were pure silica grains. Note the correlation between blurriness and length of trace. Images were digitally enhanced for presentation purposes.

where $n$ is the total number of particles along the bed surface of length $L$. Equation (1) implicitly assumes that the particles are spherical and are packed such that the grain size can be estimated in one dimension. We denote the total number of observed coarse particles as $n_{1}$. The remaining portion of the bed is assumed to be composed of a mixture of particles with a mean size equal to that of the initial bed mixture $\left(\bar{D}_{I} \cong 26\right.$ microns). Therefore, the total number of particles on the surface of the bed $n$ can be estimated from

$$
n=n_{1}+\frac{L-L_{1}}{\bar{D}_{I}}
$$

where $L_{1}$ is the total length along the observed bed surface that is composed of coarse particles. Combing equations (1) and (2) yields

$$
\bar{D}=\frac{L}{\left(n_{1}+\frac{L-L_{1}}{\bar{D}_{I}}\right)}
$$

The mean grain size of the bed surface was calculated from equation (3) where $L, L_{1}$ and $n_{1}$ were measured from the CCD images.

\section{Results}

[18] Each experiment typically lasted on the order of an hour. The bed and flow conditions were dynamic during the first $\sim 10 \mathrm{~min}$ of an experiment in which the sediment bed liquefied and then stabilized, the surface of the bed coarsened as sediment was entrained into the upper water column, and in most cases ripples formed on the bed. After about $10 \mathrm{~min}$, flows appeared to reach a quasi-steady state in which erosion and coarsening of the sediment bed ceased, suspended sediment reached a quasi-steady concentration (LDP), and ripple dimensions were roughly steady and uniform. We visually documented the transient evolution of the sediment bed during the first $10 \mathrm{~min}$ of an experiment. The quantitative measurements of particle velocities and final bed surface particle size were made after the first $\sim 20$ min of an experiment, during the quasi-steady period.

\subsection{Liquefaction}

[19] Under oscillations with $U_{\text {orb }} \geq 0.32 \mathrm{~m} / \mathrm{s}$, liquefaction of the sediment bed occurred for experiments that had newly added sediment (Table 1). The bed typically did not appear liquefied until after approximately the first 2-4 min of an experiment, after which the entire bed began to shift back and forth on the order of $10 \mathrm{~mm}$ in response to the changing pressure in the wave duct. During this time, plumes of fine sediment from $\sim 5$ to $10 \mathrm{~mm}$ below the bed surface migrated upward (Figure 5b). As these plumes reached the bed surface, the particles were rapidly entrained into the water column. During liquefaction, the bed was fluidized and did not support ripples. No significant coarsening of the bed was observed during liquefaction. The duration of liquefaction ranged from about 2 to $8 \mathrm{~min}$ and was typically the shortest (and therefore the most efficient at relieving pore pressure) for experiments with the fastest orbital velocities. Liquefaction was probably caused by heightened pore pressure within the bed [Clukey et al., 1985; Foda, 1995; Verbeek and Cornelisse, 1997]. Liquefaction ceased as this pore pressure was released due to grains shifting into more stable positions and by the loss of fine sediments from the bed which increased its permeability. After liquefaction, the bed stabilized and was rigid for the remainder of the experiment. 

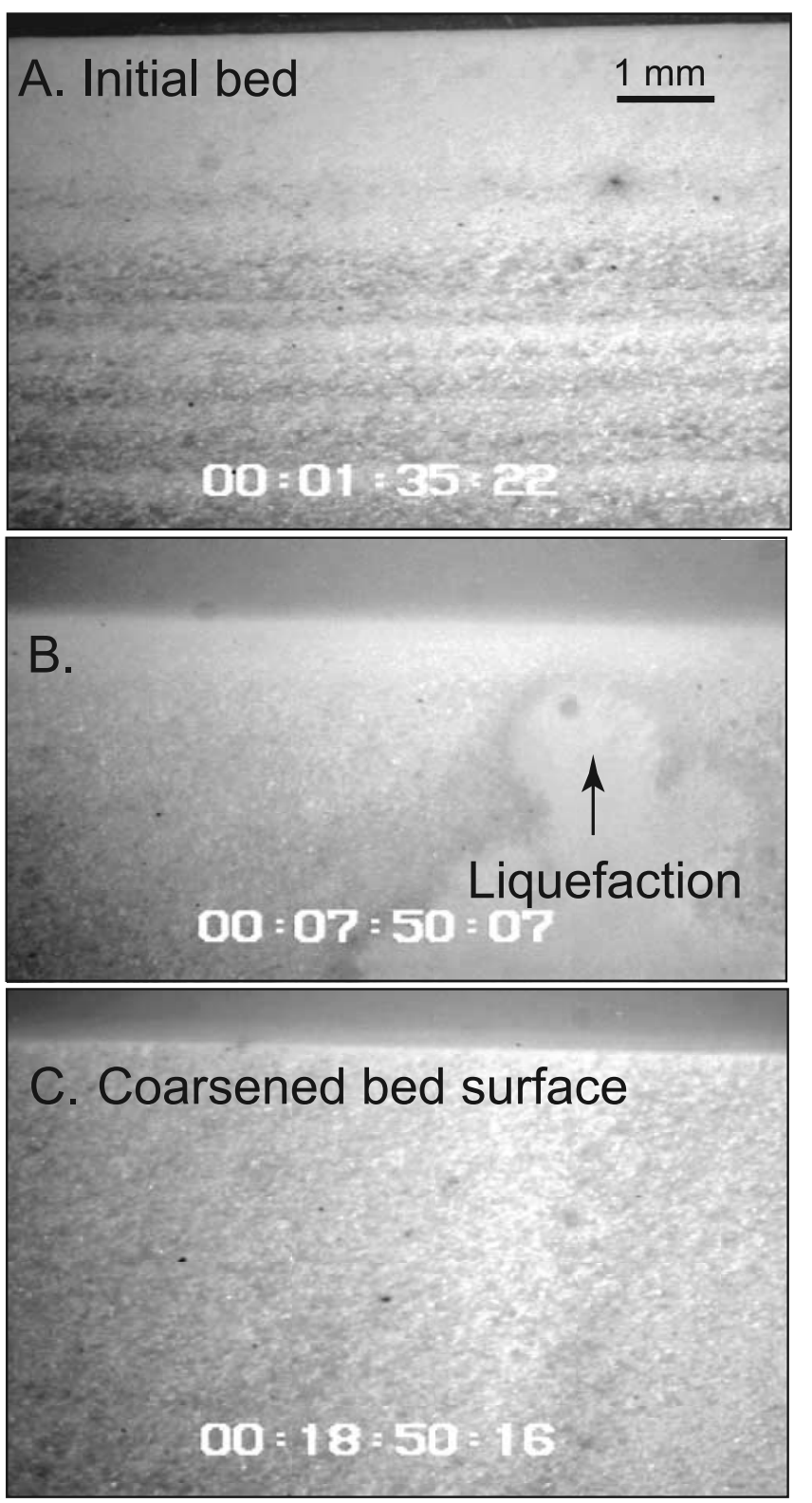

Figure 5. Photographs documenting the initial transient response of the bed to wave forcing. (a) Initial bed (run time $\sim 0$ ). (b) During liquefaction (run time $\sim 8 \mathrm{~min}$ ). (c) After liquefaction, the bed was rigid and the bed surface coarsened (run time $\sim 19 \mathrm{~min}$ ). The images were digitally enhanced for presentation purposes.

\subsection{Bed Surface Erosion and Coarsening}

[20] Significant amounts of sediment were entrained into the wave boundary-layer and the upper water-column during the first several minutes of an experiment. The bulk of this sediment was suspended within $3-8 \mathrm{~cm}$ of the bed in what have been termed high-density suspensions by LDP. For experiments with a new sediment bed, the total depth of erosion $h_{E}$ was as large as $9.5 \mathrm{~mm}$ (Table 1) (measured by visually recording the average elevation of the initial bed and $\sim 30$ min through an experiment after erosion appeared to cease). Following liquefaction, the surface of the bed rapidly coarsened as finer sediment was preferentially entrained into the water column (Figure 5c). An example of the temporal evolution of the bed is given in Figure 6a for experiment 3 , where the bed surface coarsened from 26 microns to 82 microns in about $3 \mathrm{~min}$.

[21] After this initial period of coarsening, the bed surface grain-size was relatively steady with a mean size $\bar{D}$ ranging between different experiments from 82 to 100 microns
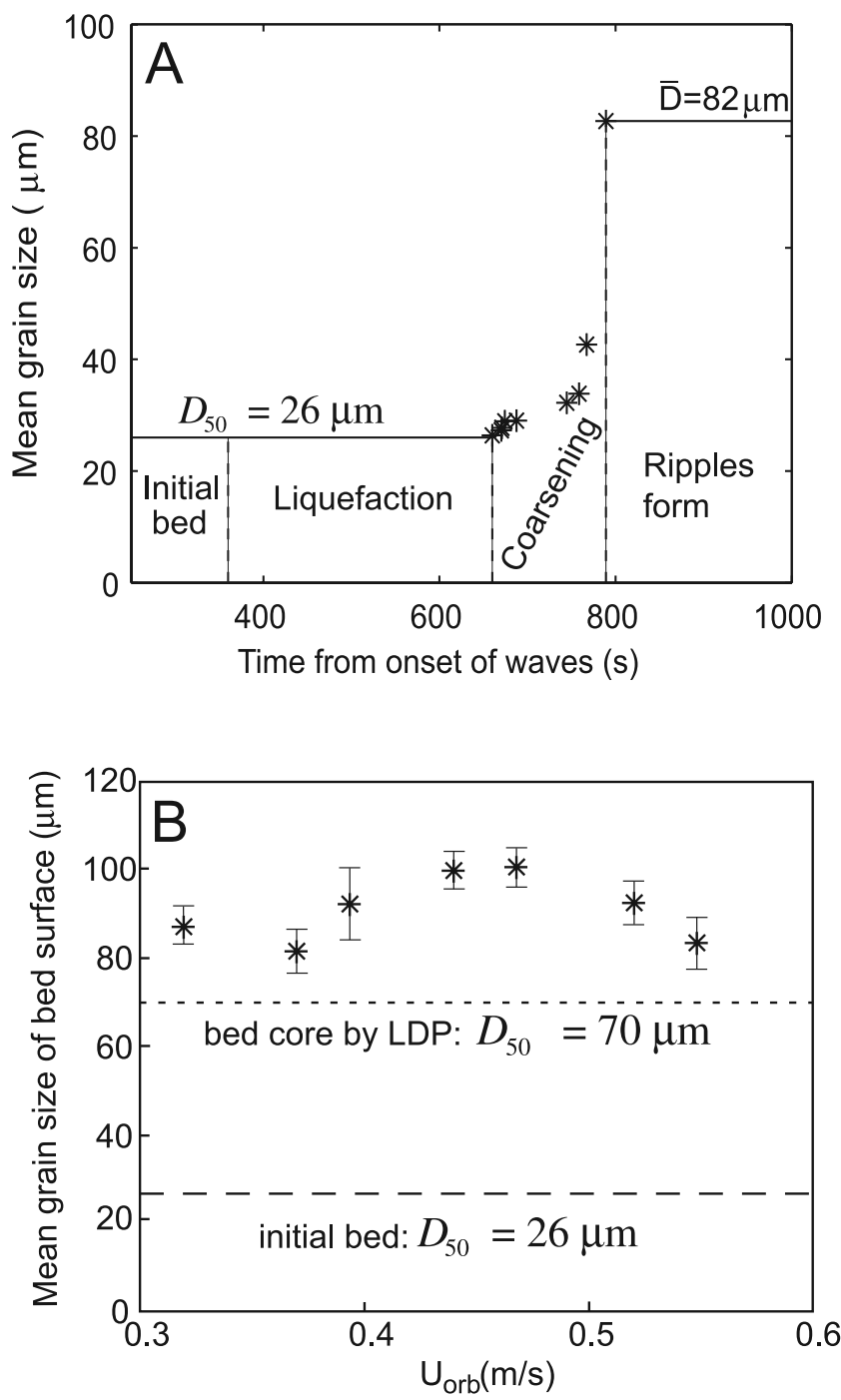

Figure 6. Direct measurements of bed surface particle size observed from micro-scale visualization. (a) Bed surface coarsening for experiment 3 . The particle size of the bed appeared to be similar to the initial bed mixture $\left(D_{50}=26 \mu \mathrm{m}\right)$ during liquefaction. After $\sim 800 \mathrm{~s}$, coarsening appeared to cease and ripples started to form on the bed surface. After about $1200 \mathrm{~s}$ (not shown), ripples reached their quasi-steady dimensions reported in Table 1 and the surface particle size remained approximately constant at $\sim 82 \mu \mathrm{m}$ for the remainder of the experiment. (b) Final, quasi-steady bedsurface particle size $\bar{D}$ for experiments $2-8$. Approximately 3-5 measurements were made at different times for each experiment during quasi-steady conditions and the error bars represent the minimum and maximum values. The bed surface size was not measured for experiment 1: it appeared to be similar to that of the initial bed. 

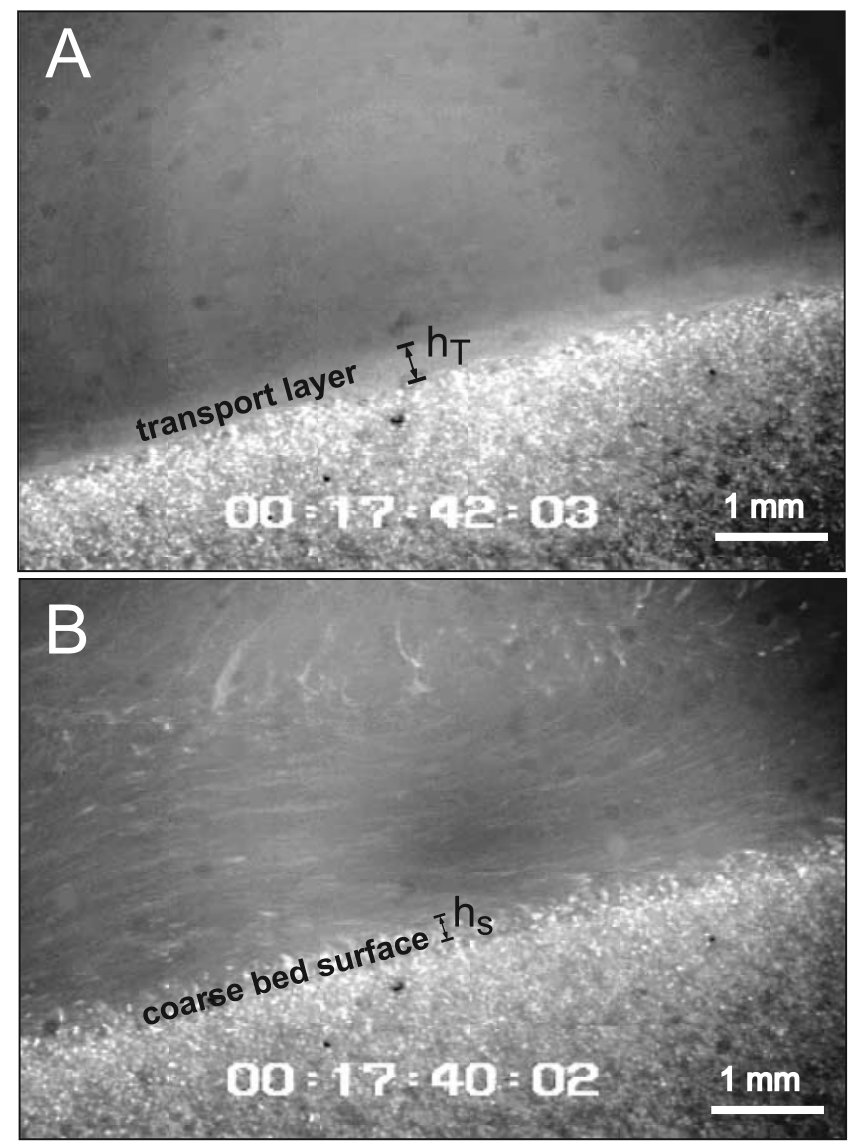

Figure 7. Micro-scale images of the bed surface from experiment 4 during (a) peak wave motion and (b) reversal of wave motion. The thickness of the near-bed transport layer $h_{T}$ and the collapsed coarse surface layer $h_{S}$ were estimated from the images (see Table 1). Images were digitally enhanced for presentation purposes.

(Table 1). Figure $6 \mathrm{~b}$ shows the bed surface particle size for each experiment, where $\bar{D}$ was averaged over $3-5$ images and the error bars define the maximum and minimum values. Coarsening of the bed surface was significant for all of the experiments, except experiment 1, which apparently was below the threshold of particle motion.

[22] Bed surface particle size was the largest for experiments with a maximum orbital velocity of about $0.45 \mathrm{~m} / \mathrm{s}$ and was smaller for experiments with both slower and faster orbital velocities (Figure 6b). The increase in coarsening with increasing wave orbital velocity probably reflects the flows ability to transport coarser grains under faster flows, leaving behind a more winnowed surface layer. However, for the strongest waves (i.e., $U_{\text {orb }} \geq 0.5 \mathrm{~m} / \mathrm{s}$ ), even the coarsest sediments were suspended, the ripples on the bed were washed out, and the size of the particles on surface of the bed was reduced.

[23] The mean size of surface sediments measured here are consistent with the value reported by $\operatorname{LDP}\left(D_{50}=70 \mu \mathrm{m}\right)$ from similar experiments (directly measured from a micro-core bed sample using a settling column), lending support for the 1D method used here to estimate particle size. The fact that our measurements indicate slightly coarser sediment than LDP is expected since their coring apparatus sampled the top several millimeters of the bed, which contained some sediment finer than that at the bed surface.

\subsection{Ripples}

[24] All of the experiments, except experiments 1 and 8, eventually produced ripples on the bed. The wave orbital velocity was too slow in experiment $1\left(U_{\text {orb }}=0.22 \mathrm{~m} / \mathrm{s}\right)$ to coarsen the bed and transport bed load. In experiment 8 , the ripples were washed out and the bed was planar. Ripple heights $\eta$ and wavelengths $\lambda$ were measured from the macroscopic images taken through the side of the flume after the ripples reached a state in which their dimensions did not significantly change in time. Ripple heights were measured from ripple troughs to crests. Approximately 10 ripples were measured for each experiment and the average values are reported in Table 1. The ripples were dimensionally similar to those reported by LDP and were suborbital or anorbital following the classification set forth by Wiberg and Harris [1994] (Table 1).

\subsection{Near-Bed Transport Layers}

[25] We observed transport of the coarse sediment on the bed surface in highly concentrated, near-bed transport layers. The thickness of the transport layers was measured from 2 or 3 CCD images per experiment for experiments 2-8. These measurements were made during quasi-steady conditions, such that there was little variation between images (typically $<10 \%$ ). The average thickness of the transport layers $h_{T}$ ranged from $0.24-0.45 \mathrm{~mm}$ for different experiments (Table 1), corresponding to about $2.5 \bar{D}-5.5 \bar{D}$. These layers consisted mostly of coarse sediment from the bed surface, which was entrained during high flow velocity (Figure 7a) and deposited as a coarse surface layer when the oscillations switched directions (Figure 7b) (i.e., flow velocity $\sim 0$ ). This process is illustrated in Figure 8 . Nearbed transport layers were observed for all of the experiments, except experiment 1 . Note that these near-bed layers should not be confused with the overlapping, less-concentrated, layer of suspended sediment that was typically $30-70 \mathrm{~mm}$ thick and was discussed in detail by LDP.

[26] We were not able to directly measure the sediment concentration within the near-bed transport layers. However, we did measure the thickness of the deposited coarse surface layers $h_{S}$ from the CCD images when the wave velocity was near zero (in the same manner as for $h_{T}$ described above). The depth-averaged volumetric concentration of suspended sediment within the near-bed transport layer $C_{T}$ then can be estimated from

$$
C_{T}=\frac{C_{S} h_{S}}{h_{T}}
$$

where $C_{S}$ is the volumetric concentration of sediment within the deposited coarsened surface layer (i.e., 1 - porosity). If we assume that $C_{S}=0.6$, then the depth-average concentration of sediment within the near-bed transport layer $C_{T}$ is calculated using equation (4) to range from 0.25 to 0.36 (Table 1). Thus, our visual measurements of the height of near-bed transport layers are consistent with previous work that has defined the layer as the region where sediment concentrations exceed $\sim 0.1$ and particle-fluid and 


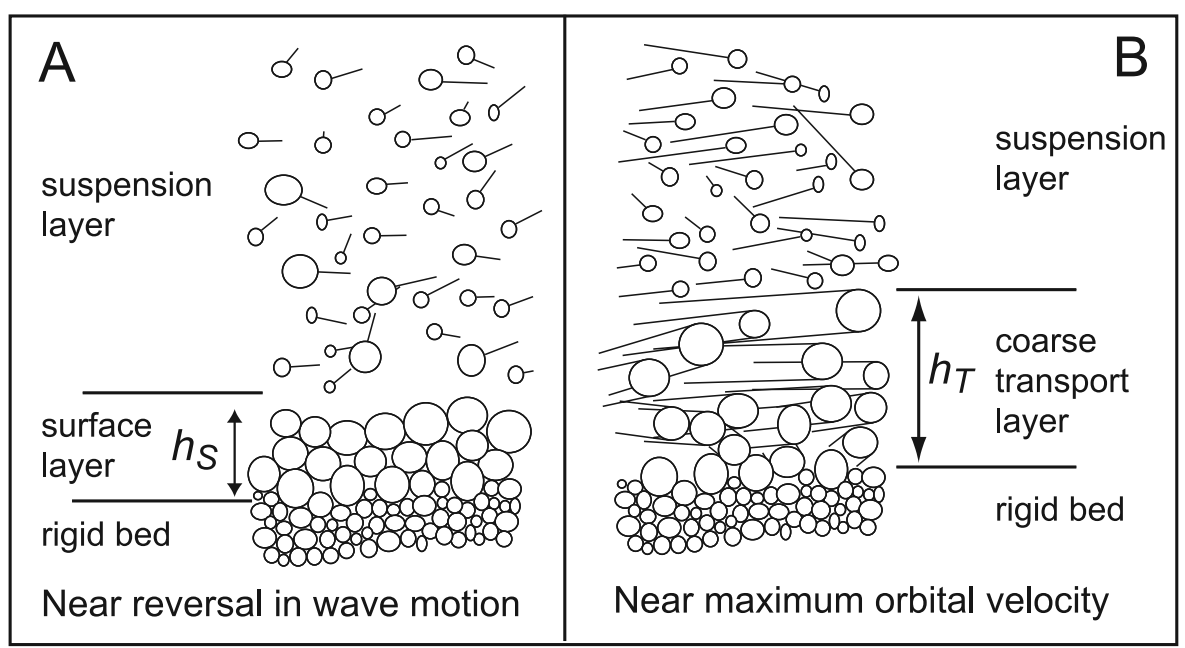

Figure 8. Cartoon illustrating (a) a collapsed coarse surface layer when the wave switched directions and the velocity was near zero and (b) a near-bed transport layer during peak velocity.

particle-particle interactions are important [e.g., DohmenJanssen et al., 2001].

[27] The near-bed transport layers comprised only a small fraction of the total amount of sediment that was eroded from the bed. The ratio of the thickness of the coarse surface layer $h_{S}$ to the depth of erosion $h_{E}$ (Table 1) ranged from 1 to $4 \%$, which is consistent with the fact that these coarse sediments were only a small faction of the original sediment mixture. The rest of the sediment was entrained into the water column and composed the high-density suspensions (LDP).

\subsection{Near-Bed Particle Velocity}

[28] During quasi-steady conditions, the CCD images were used to construct particle velocity profiles for experiments 3-8 using particle-streak velocimetry. Unfortunately, there were not enough particle tracers in any given image to construct an instantaneous velocity profile. Instead, measurements from several successive images (taken during peak velocity as described above) were overlaid to construct a profile. The profiles, therefore, represent a temporal average of maximum orbital velocity. When ripples were present, the horizontal location of the velocity profile was halfway between the trough and crest of a ripple and the measurements were taken when the flow was moving toward the ripple crest (i.e., on the stoss side of the ripple crest). The PSV measurements were consistently out of the separation zone that occurred on the lee side of the ripple crests. The ripples did not move significantly during the PSV measurements, but when ripple translation did occur, the position of the CCD camera was adjusted to maintain a position midway between the ripple trough and crest. When the bed was planar (i.e., experiment 8 ), the velocity profile was measured above the same location on the bed, near the middle of the duct.

[29] The results of the PSV measurements are shown in Figure 9. These measurements reveal a highly energetic near-bed region, with particle velocities as high as $0.2 \mathrm{~m} / \mathrm{s}$ within $1 \mathrm{~mm}$ of the immobile bed. Some of the profiles do not appear linear in log linear space, as is typical for turbulent boundary layers [Schlichting, 1968]. Instead, there appears to be a kink in some of the velocity profiles (e.g., experiments 4 and 8) at an elevation coincident with the top of the near-bed transport layer, $h_{T}$ (Figure 9). The significance of the kink is discussed in more detail below.

[30] The scatter in the measured particle velocities profiles could be due to the possibility that the local velocity was not at the peak velocity at the time of the measurement, or that the target particle was slightly within the sidewall boundary layer, as discussed in section 2.1. In addition, there was error in the vertical dimension of about plus or minus $\bar{D}$ due to the difficulty in precisely locating the elevation of the immobile bed.

\section{Analysis of Velocity Profiles}

[31] In order to analyze the velocity profiles, it is useful to calculate the roughness and bed shear velocity. The flow velocity near a boundary in steady, uniform flows is typically characterized by the "law of the wall":

$$
u=\frac{u_{*}}{\kappa} \operatorname{Ln}\left(\frac{z}{z_{0}}\right)
$$

where $\kappa$ is von Karman's constant $(\sim 0.41), u_{*}$ is the boundary shear velocity $\left(u_{*}=\sqrt{\tau_{b} / \rho}\right.$, where $\tau_{b}$ is the boundary shear stress and $\rho$ is the fluid density), and $z_{0}$ is the bed roughness parameter. Following the pioneering work of Nikuradse [1933], the flow roughness parameter is described as $z_{0}=k_{s} / 30$ for hydraulically rough flow, where $k_{s}$ is the characteristic roughness length scale of the boundary. For an immobile sediment bed of mixed particle sizes, the roughness is often described as $k_{s} \sim 2.5 \bar{D}$.

[32] The assumption of a logarithmic velocity profile is thought to be reasonable, even when there is substantial sediment transport [McEwan et al., 1999; Bauer et al., 2004] and when the flow is oscillatory [Grant and Madsen, 1982; Dohmen-Janssen et al., 2001; McLean et al., 2001]. Nonetheless, recent workers have suggested that applying equation (5) to such flows could yield errors of nearly $50 \%$ [McLean et al., 1999]. Caution must also be taken because, strictly speaking, the equation (5) only applies to the lower 

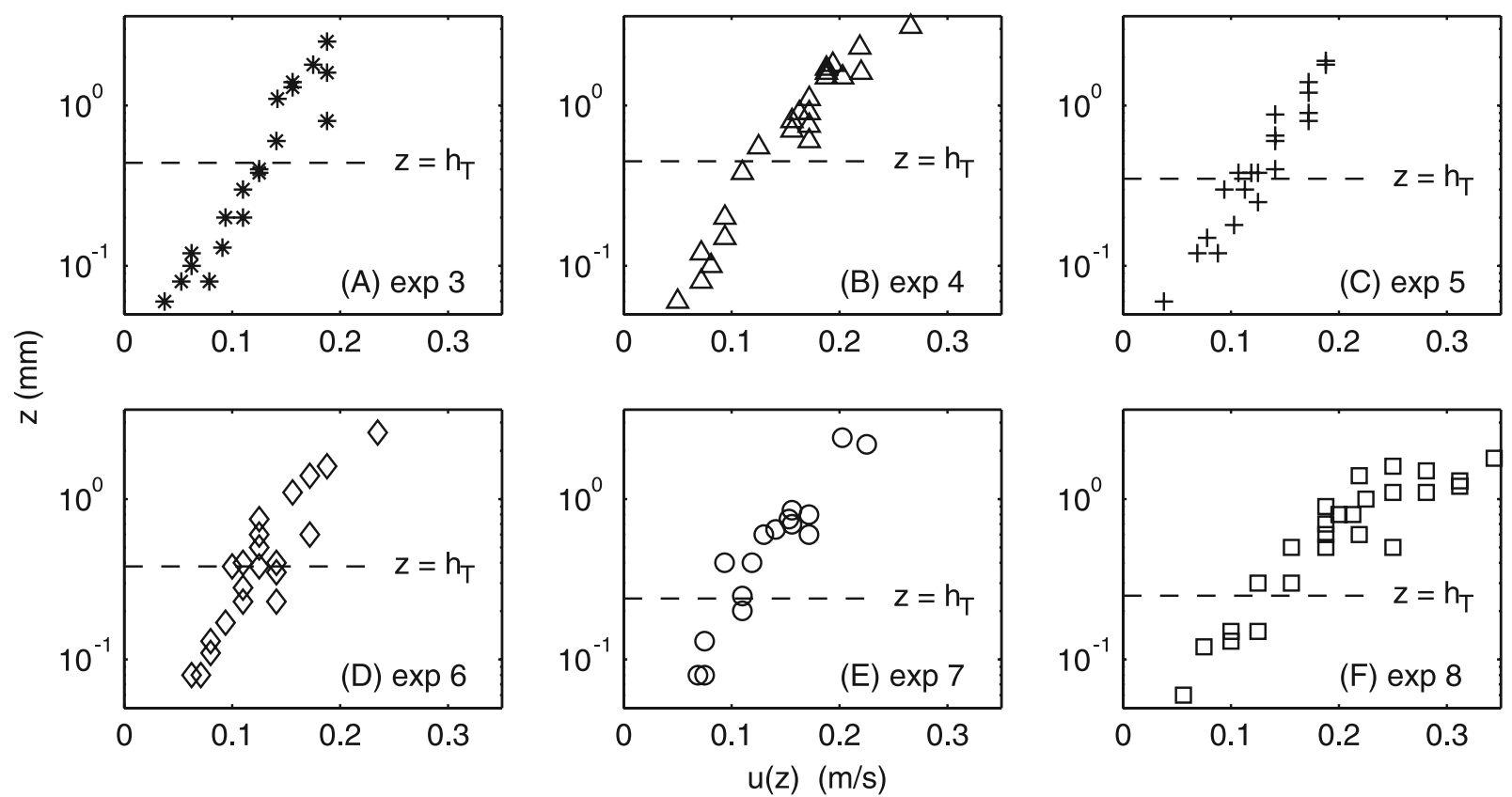

Figure 9. Maximum particle orbital velocity measured using PSV versus height above the immobile bed $(z)$ for experiments 3-8. Also shown is the observed height of the near-bed transport layers $h_{T}$.

$10-20 \%$ of the boundary layer [e.g., Grant and Madsen, 1986]. The wave boundary layers in our experiments were only on the order of $5 \mathrm{~mm}$ thick (LDP). This means that the law of the wall is only applicable to within $\sim 1 \mathrm{~mm}$ of the bed (i.e., $z / \bar{D} \leq 10$ ). Much of our data falls within this range, but there could also be overlap from the roughness layer [Campbell et al., 2005], which would further restrict the applicability of equation (5).

\subsection{Multiple Scales of Bottom Roughness}

[33] The observed kink in some of the velocity profiles (Figure 9) suggests that the flow in those experiments was affected by roughness of different scales. It is well known that such a kink can result from roughness due to near-bed sediment transport [Owen, 1964; McEwan, 1993] or bedforms [Smith and McLean, 1977] or both [Grant and Madsen, 1982]. In order to estimate the roughness parameter from each kinked profile, we fit two lines (in a leastsquared sense) to the velocity data for each experiment in log linear space: one for the data at or above $h_{T}$ (subscript $a$ ) and one for the data below $h_{T}$ (subscript $b$ ) (Figure 9). We then calculated the roughness parameter $z_{0}$ (from the y-intercept) and shear velocities $u *$ (from the slope) assuming logarithmic velocity profiles (i.e., equation (5)). This bi-linear analysis is consistent with linearly partitioning stress between multiple roughness elements [Einstein and Barbarossa, 1952; Smith and McLean, 1977; Grant and Madsen, 1982]. We used $h_{T}$ to split the data (even though it was measured independently of the velocity profiles) because the kink appeared to coincide with $h_{T}$. This allowed us to avoid an inherently subjective interpolation scheme, which would be needed to define quantitatively the elevation of the kinks.

[34] The results of this analysis indicate that the shear velocities above $h_{T}\left(u *_{a}\right)$ range from 20 to $56 \mathrm{~mm} / \mathrm{s}$ and are consistently larger than the values at the bed $\left(u_{*}\right)$ for each experiment (Table 1). The calculated roughness parameters above $h_{T}$ were found to be $z_{0 a}=0.035-0.18 \mathrm{~mm}$ (or $k_{s a} / \bar{D}=$ 11.5-66.9), while below $h_{T}, z_{0 b}=0.011-0.030 \mathrm{~mm}$ (or $k_{s b} /$ $\bar{D}=3.6-9.5)$. The shear velocities and roughness parameters above $h_{T}$ were not significantly different from those below $h_{T}$ for the experiments that did not have a well defined kink (e.g., experiment 5) (Table 1).

[35] The calculated values for the roughness parameters and shear velocities were used to collapse the experimental data, as shown in Figure 10. The velocity profiles appear to collapse when the distance from the bed is nondimensionalized by either $z_{0 a}$ or $z_{0 b}$ and the velocity is nondimensionalized by $u_{* a}$ or $u_{* b}$. Despite the scatter in the data, both methods of nondimensionalization produce a kink in the collapsed velocity profiles (Figure 10). This suggests that the apparent absence of a kink in some of the experiments (e.g., experiment 5) could be due to data resolution.

\subsection{Boundary Roughness Models}

[36] During sediment transport, energy from the mean flow energy is spent on accelerating particles and on the production of turbulent kinetic energy by wakes shed by these particles. This has the effect of increasing the effective roughness of the flow, near-bed velocity gradients, and therefore shear velocity and turbulence intensities [Best et al., 1997]. The roughness due to near-bed sediment transport is typically described by a simple formula, $k_{s} \cong \alpha h_{T}$, where $\alpha$ is an empirical constant that has been found to range from about 1 to 4 [Dietrich, 1982; Grant and Madsen, 1982; Wiberg and Rubin, 1989; Dohmen-Janssen et al., 2001]. The range in $\alpha$ might be due, in part, to the fact that roughness should also depend on the concentration of the mobile particles [McEwan et al., 1999; Bergeron and Carbonneau, 1999].

[37] Figure 11 shows the calculated roughness heights $\left(k_{s a} / \bar{D}\right.$ and $\left.k_{s b} / \bar{D}\right)$ for each experiment along with the predict 

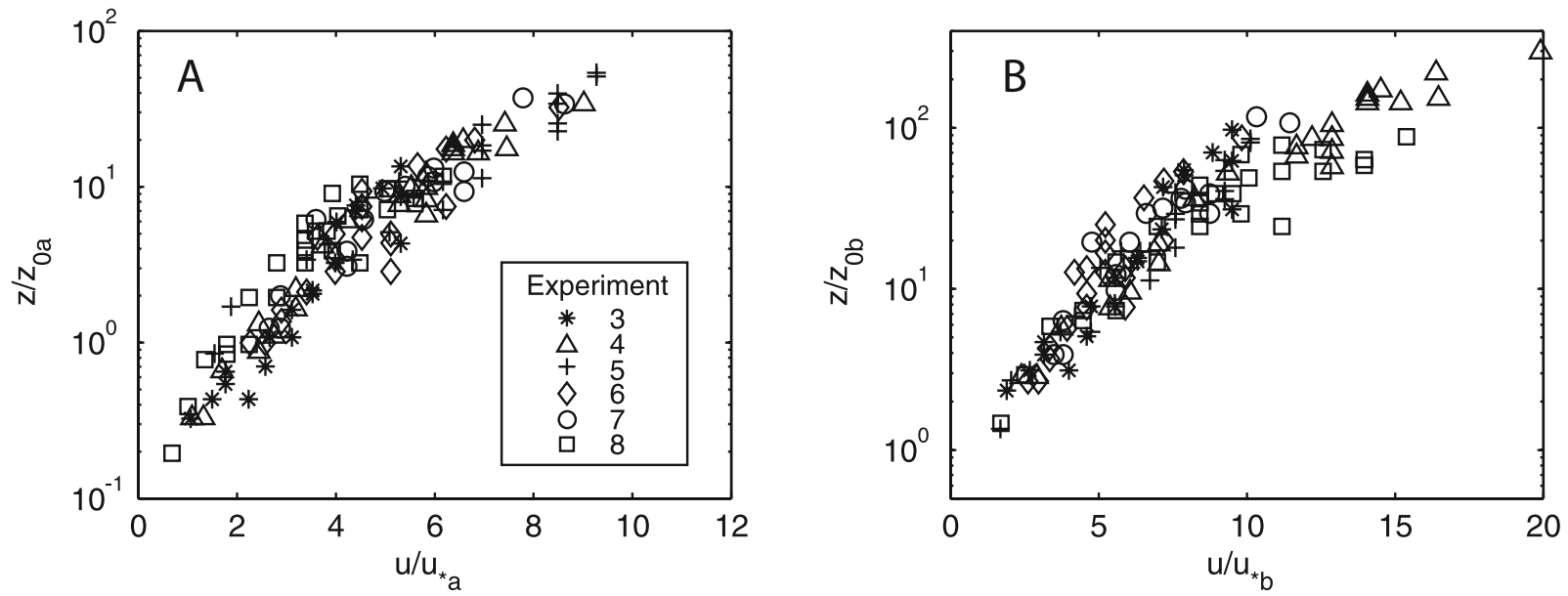

Figure 10. Velocity profiles for experiments 3-8 where the height above the bed is nondimensionalized by the roughness parameter $z_{0}$ and the velocity is nondimensionalized by the shear velocity $u *$ for the data (a) above and (b) below the height of the observed near-bed transport layers.

range of roughness for near-bed transport layers (i.e., $h_{T} \bar{D}$ to $4 h_{T} \sqrt{D}$, where $h_{T}$ is the value measured in the experiments (Table 1)). Except for experiment 5, the calculated roughness heights above $h_{T}$ (i.e., $k_{s a} / \bar{D}$ ) were consistently larger than those predicted for near-bed transport layers, which suggests that the existing near-bed transport-layer models cannot fully account for the observed roughness.

[38] It might be possible that our visual estimates of the thickness of the near-bed transport layers $h_{T}$ underestimate the actual thicknesses due to the inherent difficulty in making such measurements in the presence of significant suspended sediment. To test this, the height of the transport layer can be calculated from the formula of Dietrich [1982],

$$
\frac{h_{T}}{D}=\frac{a_{1}\left(u_{*} / u_{*_{c}}\right)^{2}}{1+a_{2}\left(u_{*} / u_{*_{c}}\right)^{2}}
$$

where $a_{1}=0.68$ and $a_{2}=0.0204(\ln D)^{2}+0.0220(\ln D)+$ 0.0709 with $D$ units in centimeters. Wiberg and Rubin [1989] found that equation (6) was a good predictor of the height of the bed-load layer based on experimental data and the saltation model of Wiberg and Smith [1985]. The predicted heights of the transport layers using equation (6) range from $h_{T} / \bar{D}=0.9$ to 1.4 for our experiments $3-8$, where we set $u_{*}=u_{*} a, u_{*_{c}}=1.2 \mathrm{~cm} / \mathrm{s}$ (as discussed below) and $D=\bar{D}$. These predicted values are smaller than the observed values $\left(h_{T} \sqrt{D}=2.5-5.5\right)$, which suggests that our visual measurements of the height of the near-bed transport layers were not underestimates.

[39] The ripples observed on the bed (for all of the experiments except experiment 8) could also contribute to the boundary roughness. Ripple-roughness length scales can be estimated as $k_{s r 1}=27.7 \eta^{2} / \lambda$ [Grant and Madsen, 1982] or $k_{s r 2}=4 \eta$ [Wikramanayake and Madsen, 1990]. Using measured values of $\eta$ and $\lambda$ from the present experiments (Table 1), the expected roughness length scales range from $0 \bar{D}$ to $292 \bar{D}$ (Figure 11). For most of the experiments, the predicted ripple roughness is greater than the observed roughness except for experiments 7 and 8 (Figure 11), which had nearly washed-out ripples and a planar bed, respectively.

[40] Comparison of the above models to our experimental measurements makes it unclear if the observed kink in the velocity profiles was due to ripples, near-bed transport layers, or both. It might be possible that the models for roughness due to ripples overestimate the measured roughness because our velocity profiles were not spatially averaged over the bedforms [Smith and McLean, 1977], as described in section 3.5. Nonetheless, the fact that the kink in the velocity profile occurred at the approximate height of

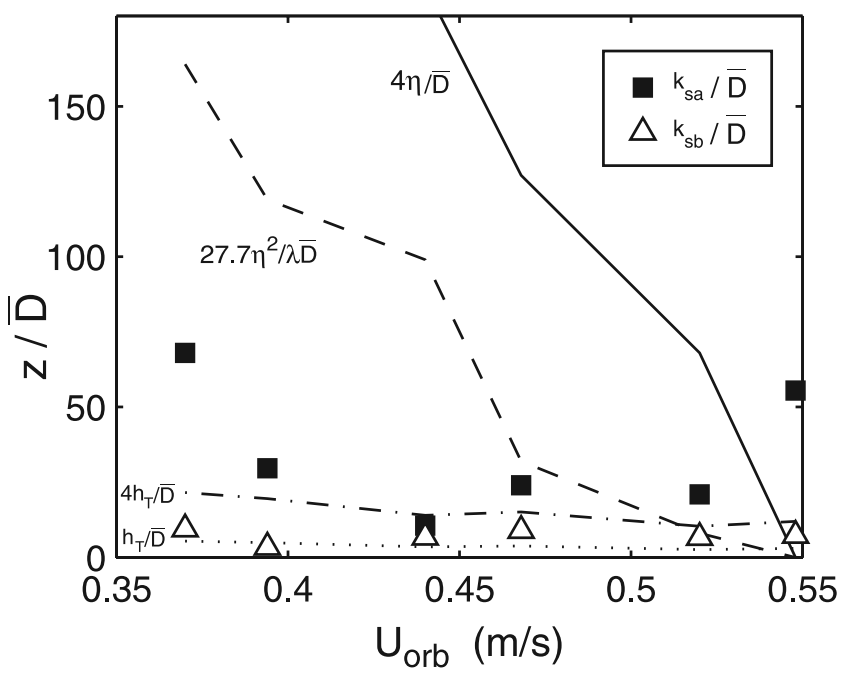

Figure 11. Calculated and predicted roughness heights. The calculated roughness heights were found from log linear fits of the measured velocity profiles for the data above $\left(k_{s a}\right)$ and below $\left(k_{s b}\right)$ the height of the near-bed transport layers. The predicted range in roughness heights for the near-bed transport layers are shown by the endmembers $h_{T}$ and $4 h_{T}$ (Table 1). Predicted roughness due to ripples were calculated from $4 \eta$ and $27.7 \eta^{2} / \lambda$, where $\eta$ denotes the average ripple heights and $\lambda$ denotes the average ripple wavelengths (Table 1). 


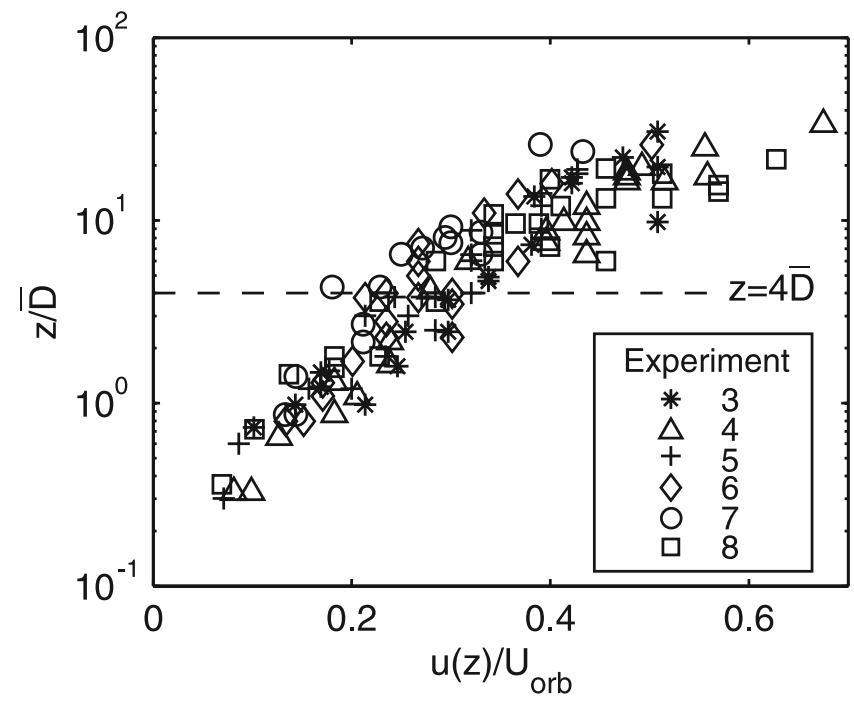

Figure 12. Velocity profiles for experiments 3-8 where the height above the bed is nondimensionalized by the mean bed-surface particle size $\bar{D}$ and the velocity is nondimensionalized by the maximum free-stream orbital velocity $U_{\text {orb }}$.

the near-bed transport layers, suggests that the heightened roughness was due to the transport layer [Owen, 1964; McEwan, 1993]. The location of the kink is thought to relate to the height at which the sediment extracts the most momentum from the flow [Bagnold, 1941]. The ripples crests were at an elevation of about $0.5 \eta$ above the immobile bed at the location of the velocity profile (i.e., halfway between ripple trough and crest), or about $25 \bar{D}$ (Table 1), which indicates that the ripple crests were well above the kink in the velocity profile. Moreover, ripples did not occur in experiment 8 and experiment 8 had one of the most obvious kinks in the velocity profiles. Ripples, when present, are typically thought to dominate hydraulic roughness [Grant and Madsen, 1982]. Our experiments suggest that this might not always be the case and that hydraulic roughness from near-bed transport layers might be more important when ripples are present than previously thought. This is consistent with the observations of LDP that the ripples appeared to offer little roughness to the high-density suspensions. Instead the suspensions appeared to smoothly move over the ripples with a constant thickness, accurately reflecting the topography of the bed. This might be particularly true in these experiments because sediment-induced stratification limited vertical mixing of momentum and reduced the boundary layer height in comparison to comparable clear water flows (LDP). The ripples might not have acted as significant roughness elements to the developing boundary layers, since the boundary layers were typically smaller than the ripple heights. Dohmen-Janssen et al. [2001] also note that they were unable to correctly predict observed velocity profiles above sandy sheet flows without both a roughness correction due to the sheet flows and a stratification correction due to the entrained sediment.

\subsection{Bed Stress and Incipient Motion}

[41] One of the proposed implications of near-bed transport layers is that, under equilibrium sediment-transport conditions, the momentum deficit should reduce the available fluid stress at the bed to the critical stress necessary for incipient particle motion [Bagnold, 1956]. The threshold of motion can be calculated from $\theta_{c}=u_{*_{c}}^{2} / r g \bar{D}$ [Shields, 1936], where $r$ is the submerged specific density of the sediment $(\sim 1.65)$ and $g$ is the gravitation acceleration. $\theta_{c}$ depends on the particle Reynolds number, $\operatorname{Re} e_{p}=\sqrt{\operatorname{rg} \bar{D}} \bar{D} / \nu$, where $\nu$ is the kinematics viscosity of the fluid. Using the empirical equation of Brownlie [1981], we calculated the critical shear velocity $u_{*_{c}} \sim 1.2 \mathrm{~cm} / \mathrm{s}$ for experiments $3-8$. Our measured values $\left(u_{* b}=1.3-2.4 \mathrm{~cm} / \mathrm{s}\right.$, Table 1$)$ for these experiments are close to, but larger than calculated value for incipient motion. This could simply be a result of the highly unsteady flow and sediment transport in the present experiments. Alternatively, these results might indicate that the dynamic boundary condition proposed by Bagnold [1956] should be abandoned in favor of a dynamic equilibrium between deposition and entrainment of grains as proposed by Einstein [1950] (see Seminara et al. [2002] and Parker et al. [2003] for a review of these hypotheses). Alternatively, the stress on the bed might have been greater than the critical stress for incipient motion because of additional roughness, for example, due to wakes shed by rolling or slowly moving grains [Whiting and Dietrich, 1990]. This latter possibility is supported by the fact that the near-bed roughness $k_{s b} / \bar{D}$ ranged from 3.5 to 9.5 (Table 1), which is greater than the typical value used for grain-induced skin friction of $k_{S} / \bar{D}=1$ to 3 [Nikuradse, 1933].

\subsection{Height of the Boundary Layer}

[42] LDP showed that the wave boundary layer was reduced in size in the presence of suspended sediment as compared to sediment-free conditions, from a few centimeters to a few millimeters thick. They questioned these findings, however, because they were not able to measure the flow velocity within $3 \mathrm{~mm}$ of the bed (due to instrumentation constraints) and the validity of acoustic measurements using an ADV in a highly turbid flow is unclear. Unfortunately, our measurements do not extend to the top of the wave boundary layer (i.e., where $u \approx U_{\text {orb }}$ ), so we cannot robustly validate the measurements of LDP. We can, however, estimate the height of the boundary layers by nondimensionalizing the distance from the bed by $\bar{D}$ and the velocity by $U_{\text {orb }}$ (Figure 12). Note that the collapsed velocity profiles in Figure 12 have a kink at about $z / \bar{D}=4$, which is consistent with the height of the near-bed transport layers $\left(h_{T} / \bar{D}=2.5-5.5\right)$, lending further support for the bilinear fit used in section 4.1. We fit a line (in log linear space, as in section 4.1) to the collapsed data for $z / \bar{D}>4$ in Figure 12 and extrapolated to estimate the height of the wave boundary layer (i.e., $z / \bar{D}$ where $u=U_{\text {orb }}$ ). This resulted in an estimated wave boundary layer height of $145 \bar{D}$, or about $1.5 \mathrm{~cm}$. This indicates that the boundary layer reduction due to sediment stratification might not be as profound as that deduced by LDP.

\section{Discussion of Results}

[43] Ripples were observed in nearly all of our experiments. Though ripples are traditionally associated with sandy sediments, ripples have been recently observed in predominantly muddy environments [i.e., Nakayama, 
2003]. The surface coarsening observed in our experiments occurred for length scales $(<1 \mathrm{~mm})$ much smaller than can be seen in x-radiographs or sampled using traditional sedimentological tools [e.g., Mullenbach and Nittrouer, 2000; Lomnicky et al., 2006]. It is not surprising, then, that the features described herein have not been described in depth before.

[44] With that said, microfabric analysis of inner-shelf cores in sedimentologically active, muddy environments has been performed. For example, Kuehl et al. [1988] observed micro-lenses of coarse sediment (1-5D thick) on the Amazon shelf that appear similar to the coarse lenses seen in our micro-scale visualizations. Because their observations were made in an environment with high sedimentation rates, the rapid deposition of incoming fine-grained material preserved the coarsened surfaces. Aside from the presence of substantial sediment input, the physical environment in which these observations were made was similar to the conditions examined in this study (i.e., the typical wave period is $5-10 \mathrm{~s}$ on Amazon and $3-7 \mathrm{~s}$ in this laboratory study), lending credence to the capability of the experimental wave duct to simulate natural processes at field scale.

[45] Sheet flows have been recognized to be important transport processes in sandy nearshore environments for more than 20 years [e.g., Hanes and Bowen, 1985; Dohmen-Janssen and Hanes, 2002; Hsu and Hanes, 2004]. Some observations of sheet flows in experiments and models are consistent with the near-bed transport layers observed in our experiments, despite the fact that these studies typically used all sand-sized particles and plane-bed conditions (i.e., no ripples). For example, using a dynamic two-phase model, Hsu and Hanes [2004] described a thin (20D), highly concentrated $(0.1-0.6)$ and quickly moving $\left(u \approx U_{\text {orb }} / 3\right.$ ) sheet flow during peak wave motion. To our knowledge, the development of sandy near-bed transport layers from muddy beds has not been discussed before. In the present experiments, only a few percent of the initial bed participated in nearbed transport layers. This suggests that dramatic near-bed coarsening can lead to the development of sandy near-bed transport layers during energetic storm conditions even when the sediment bed contains a very small fraction of sand, perhaps a few percent or less. The commonality of the features in these radically different settings argues that near-bed transport layers are likely more common and important than previously thought.

[46] It should be noted that some of the winnowing in the present experiments was due to entrainment of fine sediment into the upper water column and end tanks due to unavoidable background turbulence in the experimental duct. In an environment without background turbulence (e.g., due to tidal or storm currents), winnowing of the bed might not be as profound as observed in these experiments. However, even in this case some winnowing should still occur as much of sediment eroded from the bed in these experiments was entrained by turbulence generated at or near the bed by natural boundary layer processes (LDP).

[47] The calculations in section 4 of the hydraulic roughness and fluid stress from the velocity profiles implicitly assume that the particles provided an accurate tracer of the flow. It is possible, however, that the particles lagged the flow, especially within transport layer where particles sizes were relatively large and sediment concentrations were high [Best et al., 1997; McEwan et al., 1999; Cheng, 2004]. Accounting for such a lag could reduce or perhaps eliminate the observed kink in the velocity profiles. Further experiments using techniques capable of simultaneously measuring fluid and particle velocities are needed to explore this issue.

\section{Summary and Conclusions}

[48] We report on experiments of oscillatory flow over a predominantly silty sediment bed. The sediment bed was highly dynamic during the first several minutes of wave forcing. Liquefaction allowed plumes of material within the bed to rise to the bed surface. Fine sediment was rapidly entrained into the water column and coarse sediment was left as a sandy lag at or near the bed surface. Despite only composing a few percent of the initial bed, these coarser sediments had a profound effect on flow hydraulics and sediment transport. These coarse sediments moved as nearbed transport layers that appeared similar to sheet flows observed on sandy planar beds. Under relatively low wave orbital velocities, these transport layers contributed to the development of sandy sub-orbital or anorbital ripples on an otherwise muddy bed. At high orbital velocities, ripples were washed out and the bed was planar.

[49] Detailed particle-streak velocimetry revealed that the velocity profiles were kinked in log linear space suggesting multiple scales of hydraulic boundary roughness. Ripples, near-bed transport layers, or both, could have contributed to boundary roughness and caused the observed kinks in the velocity profiles. Roughness models for both ripples and near-bed sediment transport, however, provided rather poor predictions of the roughness heights from the measured velocity profiles. Formation of the kink by near-bed sediment transport is supported by the fact that the kink was observed when ripples were not present (e.g., experiment 8) and that the elevation of the kink coincided with the observed height of the near-bed transport layers. Furthermore, heightened roughness by these near-bed transport layers is consistent with the measurements in similar experiments by LDP of reduced mixing lengths and increased turbulent kinetic energy in the extreme near-bed region.

[50] Near-bed transport layers could cause significant transport of sand, even in muddy environments. They also likely limit entrainment of the underlying finer grains by armoring the bed and reducing the stress at the bed available to entrain sediment. Future modeling efforts of sediment transport on fine-grained continental shelves should consider liquefaction, bed surface coarsening, ripple formation, stratification by suspended and bed-load sediment, and heightened roughness and production of turbulent kinetic energy from near-bed transport layers within wave boundary layers.

\section{Notation}

$C_{S} \quad$ volume concentration of sediments in the porous bed surface layer.

$C_{T}$ volume concentration of sediments in the nearbed transport layer.

$D$ sediment grain size. 
$\bar{D}$ mean grain size of bed surface.

$\bar{D}_{I}$ mean grain size of the initial bed.

$D_{50}$ median grain size.

$d_{0}$ wave orbital diameter.

GM Grant and Madsen [1979, 1982, 1986].

$g$ gravitational acceleration $\left(=9.81 \mathrm{~m} / \mathrm{s}^{2}\right)$.

$h_{E}$ total eroded depth of bed.

$h_{T}$ thickness of the near-bed transport layer.

$h_{S}$ thickness of the coarsened surface layer.

$k_{s}$ roughness length scale.

$k_{s a} \quad$ calculated roughness for data above $h_{T}$.

$k_{s b} \quad$ calculated roughness for data below $h_{T}$.

$k_{s r 1}$ boundary roughness due to ripples [Grant and Madsen, 1982].

$k_{s r 2}$ boundary roughness due to ripples [Wikramanayake and Madsen, 1990].

LPD Lamb et al. [2004]; Lamb and Parsons [2005].

$R e_{p}$ particle Reynolds number.

$r$ submerged specific density of sediment $(\sim 1.65)$.

$T$ wave period (s).

$U_{\text {orb }}$ free-stream maximum wave-orbital velocity.

$u$ velocity.

$u_{*}$ shear velocity.

$u_{*} \quad$ calculated shear velocity for data above $h_{T}$.

$u_{* b} \quad$ calculated shear velocity for data below $h_{T}$.

$u_{*_{c}}$ critical shear velocity for incipient particle motion.

$z$ distance above the immobile bed.

$z_{0}$ roughness parameter $\left(=k_{s} / 30\right)$.

$\alpha$ coefficient relating $h_{T}$ to $k_{s}$.

$\eta$ ripple height.

$\theta_{c}$ critical Shields parameter for incipient particle motion.

$\kappa \quad$ von Karman's constant $(=0.41)$.

$\lambda$ ripple wavelength.

$\nu$ kinematic viscosity $\left(\sim 1 \mathrm{~mm}^{2} / \mathrm{s}\right)$.

$\rho$ fluid density $\left(\sim 1000 \mathrm{~kg} / \mathrm{m}^{3}\right)$.

$\tau_{b}$ boundary shear stress.

[51] Acknowledgments. Financial support for the work performed was provided by grants from ONR (N00014-03-10138), NSF (EAR0309887), and the American Chemical Society Petroleum Research Fund. The authors wish to thank T. Maxworthy and A. M. Fincham for lending us the optical system.

\section{References}

Anderson, R. S., and B. Hallet (1986), Sediment transport by windToward a general model, Geol. Soc. Am. Bull., 97(5), 523-535.

Bagnold, R. A. (1941), The Physics of Blown Sand and Desert Dunes, 265 pp., Methuen, New York.

Bagnold, R. A. (1956), The flow of cohesionless grains in fluids, Philos. Trans. R. Soc. London A, 249(964), 235.

Bauer, B. O., C. A. Houser, and W. G. Nickling (2004), Analysis of velocity profile measurements from wind-tunnel experiments with saltation, Geomorphology, 59(1-4), 81-98.

Bergeron, N. E., and P. Carbonneau (1999), The effect of sediment concentration on bedload roughness, Hydrol. Proc., 13(16), 2583-2589.

Best, J., S. Bennett, J. Bridge, and M. Leeder (1997), Turbulence modulation and particle velocities over flat sand beds at low transport rates, J. Hydraul. Eng., 123(12), 1118-1129.

Brownlie, W. R. (1981), Prediction of flow depth and sediment discharge in open channels, Rep. KH-R-43A, 232 pp., W. M. Keck Lab. of Hydraul. and Water Resour., Calif. Inst. of Technol., Pasadena.

Campbell, L., I. McEwan, V. Nikora, D. Pokrajac, M. Gallagher, and C. Manes (2005), Bed-load effects on hydrodynamics of rough-bed open-channel flows, J. Hydraul. Eng., 131(7), 576-585.

Cheng, N. S. (2004), Analysis of velocity lag in sediment-laden open channel flows, J. Hydraul. Eng., 130(7), 657-666.
Clukey, E. C., F. H. Kulhawy, P. L. F. Liu, and G. B. Tate (1985), The impact of wave loads and pore-water pressure generation on initiation of sediment transport, Geo Mar. Lett., 5(3), 177-183.

Crockett, J. S., and C. A. Nittrouer (2004), The sandy inner shelf as a repository for muddy sediment: An example from northern California, Cont. Shelf Res., 24(1), 55-73.

Dietrich, W. E. (1982), Flow, boundary shear stress, and sediment transport in a river meander, Ph.D. thesis, Univ. of Wash., Seattle.

Dohmen-Janssen, C. M., and D. M. Hanes (2002), Sheet flow dynamics under monochromatic nonbreaking waves, J. Geophys. Res., 107(C10), 3149, doi:10.1029/2001JC001045.

Dohmen-Janssen, C. M., W. N. Hassan, and J. S. Ribberink (2001), Mobilebed effects in oscillatory sheet flow, J. Geophys. Res., 106(C11), $27,103-27,115$.

Einstein, H. A. (1950), The bedload function for sediment transportation in open channel flow, Tech. Bull. U. S. Dep. Agric., 1026.

Einstein, H. A., and N. L. Barbarossa (1952), River channel roughness, Trans.Am. Soc. Civ. Eng., 117, 1121-1132.

Flores, N. Z., and J. F. A. Sleath (1998), Mobile layer in oscillatory sheet flow, J. Geophys. Res., 103(C6), 12,783-12,793.

Foda, M. A. (1995), Sea floor dynamics, in Proceedings of the Advances in Coastal and Ocean Engineering, vol. 1, edited by P. L.-F. Liu, pp. $77-$ 124, World Sci., Hackensack, N. J.

Foda, M. A. (2003), Role of wave pressure in bedload sediment transport, J. Waterw. Port Coastal Ocean Eng., 129(6), 243-249.

García, M., and G. Parker (1991), Entrainment of bed sediment into suspension, J. Hydraul. Eng., 117(4), 414-435.

Glenn, S. M., and W. D. Grant (1987), A suspended sediment stratification correction for combined wave and current flows, J. Geophys. Res., 92(C8), 8244-8264.

Grant, W. D., and O. S. Madsen (1979), Combined wave and current interaction with a rough bottom, J. Geophys. Res., 84(C4), 17971808

Grant, W. D., and O. S. Madsen (1982), Movable bed roughness in unsteady oscillatory flow, J. Geophys. Res., 87(C1), 469-481.

Grant, W. D., and O. S. Madsen (1986), The continental-shelf bottom boundary-layer, Ann. Rev. Fluid Mech., 18, 265-305.

Gust, G., and J. B. Southard (1983), Effects of weak bed-load on the universal law of the wall, J. Geophys. Res., 88(C10), 5939-5952.

Hanes, D. M., and A. J. Bowen (1985), A granular-fluid model for steady intense bed-load transport, J. Geophys. Res., 90(C5), 9149-9158.

Hsu, T. J., and D. M. Hanes (2004), Effects of wave shape on sheet flow sediment transport, J. Geophys. Res., 109, C05025, doi:10.1029/ 2003JC002075.

Kuehl, S. A., C. A. Nittrouer, and D. J. DeMaster (1988), Microfabric study of fine-grained sediments - Observations from the Amazon subaqueous delta, J. Sediment. Petrol., 58(1), $12-23$.

Lamb, M. P., and J. D. Parsons (2005), High-density suspensions formed under waves, J. Sediment. Res., 75(3), 386-397.

Lamb, M. P., E. A. D'Asaro, and J. D. Parsons (2004), Turbulent structure of high-density suspensions formed under waves, J. Geophys. Res., 109 , C12026, doi:10.1029/2004JC002355.

Lomnicky, T. D., C. A. Nittrouer, and B. L. Mullenbach (2006), Impact of local morphology on sedimentation in a submarine canyon, ROV studies in Eel Canyon, J. Sediment. Res, in press.

McEwan, I. K. (1993), Bagnold's kink - a physical feature of a wind velocity profile modified by blown sand, Earth Surf. Processes Landforms, $18(2), 145-156$

McEwan, I. K., B. J. Jefcoate, and B. B. Willetts (1999), The grain-fluid interaction as a self-stabilizing mechanism in fluvial bed load transport, Sedimentology, 46(3), 407-416.

McLean, S. R., S. R. Wolfe, and J. M. Nelson (1999), Spatially averaged flow over a wavy boundary revisited, J. Geophys. Res., 104(C7), $15,743-15,753$

McLean, S. R., J. S. Ribberink, C. M. Dohmen-Janssen, and W. N. Hassan (2001), Sand transport in oscillatory sheet flow with mean current, J. Waterw. Port Coastal Ocean Eng., 127(3), 141-151.

Mullenbach, B. L., and C. A. Nittrouer (2000), Rapid deposition of fluvial sediment in the Eel Canyon, northern California, Cont. Shelf Res., 20(16), $2191-2212$

Nakayama, J. S. (2003), Time-series monitoring of bottom roughness on the northern California continental shelf, M. S. thesis, Univ. of Wash., Seattle.

Nikuradse, J. (1933), Stromungsgesetze in rauhen Rohren, Forschg. Arb. Ing. Wes., 361, 22.

Ogston, A. S., D. A. Cacchione, R. W. Sternberg, and G. C. Kineke (2000), Observations of storm and river flood-driven sediment transport on the northern California continental shelf, Cont. Shelf Res., 20, 2141-2162. Owen, P. R. (1964), Saltation of uniform grains in air, J. Fluid Mech., 20(2), $225-242$. 
Parker, G., G. Seminara, and L. Solari (2003), Bed load at low Shields stress on arbitrarily sloping beds: Alternate entrainment formulation, Water Resour. Res., 39(7), 1183, doi:10.1029/2001WR001253.

Schlichting, H. (1968), Boundary-Layer Theory, 748 pp., McGraw-Hill, New York.

Scully, M. E., C. T. Friedrichs, and L. D. Wright (2003), Numerical modeling of gravity-driven sediment transport and deposition on an energetic continental shelf: Eel River, northern California, J. Geophys. Res., 108(C4), 3120, doi:10.1029/2002JC001467.

Seminara, G., L. Solari, and G. Parker (2002), Bed load at low Shields stress on arbitrarily sloping beds: Failure of the Bagnold hypothesis, Water Resour. Res., 38(11), 1249, doi:10.1029/2001WR000681.

Shields, A. (1936), Awendung der Aehnlichkeitsmechanik und der Turbulenzforschung auf die Geschiebebewegung, Mitt. Preuss. Versuchsanst. Wasserbau Schiffbau, 26, 26.

Smith, J. D., and S. R. McLean (1977), Spatially averaged flow over a wavy surface, J. Geophys. Res., 82(12), 1735-1746.

Styles, R., and S. M. Glenn (2000), Modeling stratified wave and current bottom boundary layers on the continental shelf, J. Geophys. Res., 105(C10), 24,119-24,139.

Styles, R., and S. M. Glenn (2005), Long-term sediment mobilization at sandy inner shelf site, LEO-15, J. Geophys. Res., 110, C04S90, doi:10.1029/2003JC002175.

Traykovski, P., W. R. Geyer, J. D. Irish, and J. F. Lynch (2000), The role of wave-induced density-driven fluid mud flows for cross-shelf transport on the Eel River continental shelf, Cont. Shelf Res., 20, 2113-2140.

Verbeek, H., and J. M. Cornelisse (1997), Erosion and liquefaction of natura mud under surface waves, in Cohesive Sediments, edited by N. Burt, R. Parker, and J. Watts, pp. 353-363, John Wiley, Hoboken, N. J.

Whiting, P. J., and W. E. Dietrich (1990), Boundary shear-stress and roughness over mobile alluvial beds, J. Hydraul. Eng., 116(12), 1495-1511.
Wiberg, P. L., and C. K. Harris (1994), Ripple geometry in wave dominated environments, J. Geophys. Res., 99(C1), 775-789.

Wiberg, P. L., and D. M. Rubin (1989), Bed roughness produced by saltating sediment, J. Geophys. Res., 94(C4), 5011-5016.

Wiberg, P. L., and J. D. Smith (1983), A comparison of field data and theoretical models for wave-current interactions at the bed on the continental shelf, Cont. Shelf Res., 2, 147-162.

Wiberg, P. L., and J. D. Smith (1985), A theoretical model for saltating grains in water, J. Geophys. Res., 90(C4), 7341-7354.

Wikramanayake, P. N., and O. S. Madsen (1990), Calculation of movable bed friction factors, Tech. Rep. DACW-39-88-K-0047, 105 pp., U. S Army Corps of Eng., Coastal Eng. Res. Cent., Vicksburg, Miss.

Wright, L. D., and C. A. Nittrouer (1995), Dispersal of river sediments in coastal seas-6 contrasting cases, Estuaries, 18(3), 494-508.

Wright, L. D., C. T. Friedrichs, S. C. Kim, and M. E. Scully (2001), Effects of ambient currents and waves on gravity-driven sediment transport on continental shelves, Mar. Geol., 175(1-4), 25-45.

Wright, L. D., C. T. Friedrichs, and M. E. Scully (2002), Pulsational gravity-driven sediment transport on two energetic shelves, Cont. Shelf Res., 22(17), 2443-2460.

M. P. Lamb, Department of Earth and Planetary Sciences, University of California, Berkeley, CA 94720, USA.

H. Liang, Applied Physics Laboratory, University of Washington, Seattle, WA 98105, USA. (hanzhuan@apl.washington.edu)

J. D. Parsons, School of Oceanography, University of Washington, Seattle, WA 98195, USA 